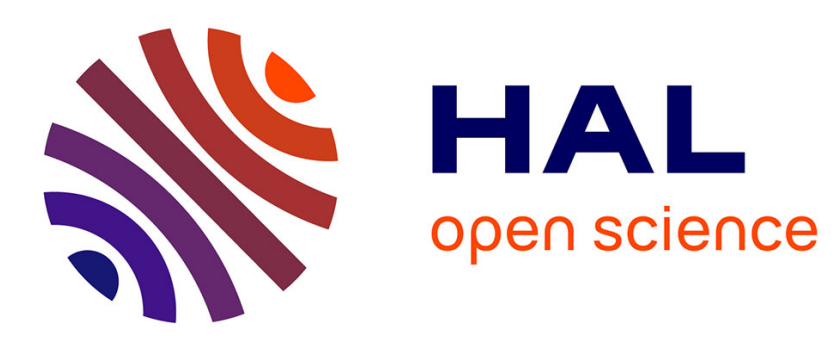

\title{
La référence au Vietnam et l'émergence des gauches radicales au Liban, 1962-1976
}

Laure Guirguis

\section{To cite this version:}

Laure Guirguis. La référence au Vietnam et l'émergence des gauches radicales au Liban, 1962-1976. Monde(s). Histoire, Espaces, Relations, 2018, 14, pp.223-242. 10.3917/mond1.182.0223 . hal02327787

\section{HAL Id: hal-02327787 https://hal-amu.archives-ouvertes.fr/hal-02327787}

Submitted on 23 Oct 2019

HAL is a multi-disciplinary open access archive for the deposit and dissemination of scientific research documents, whether they are published or not. The documents may come from teaching and research institutions in France or abroad, or from public or private research centers.
L'archive ouverte pluridisciplinaire HAL, est destinée au dépôt et à la diffusion de documents scientifiques de niveau recherche, publiés ou non, émanant des établissements d'enseignement et de recherche français ou étrangers, des laboratoires publics ou privés. 


\section{LA RÉFÉRENCE AU VIETNAM ET L'ÉMERGENCE DES GAUCHES RADICALES AU LIBAN, 1962-1976}

Laure Guirguis

Presses universitaires de Rennes | «Monde(s)»

2018/2 N 14 | pages 223 à 242

ISSN 2261-6268

ISBN 9782753575776

Article disponible en ligne à l'adresse :

https://www.cairn.info/revue-mondes-2018-2-page-223.htm

Distribution électronique Cairn.info pour Presses universitaires de Rennes.

(C) Presses universitaires de Rennes. Tous droits réservés pour tous pays.

La reproduction ou représentation de cet article, notamment par photocopie, n'est autorisée que dans les limites des conditions générales d'utilisation du site ou, le cas échéant, des conditions générales de la licence souscrite par votre établissement. Toute autre reproduction ou représentation, en tout ou partie, sous quelque forme et de quelque manière que ce soit, est interdite sauf accord préalable et écrit de l'éditeur, en dehors des cas prévus par la législation en vigueur en France. Il est précisé que son stockage dans une base de données est également interdit. 


\section{La référence au Vietnam et l'émergence des gauches radicales au Liban, 1962-1976}

Laure Guirguis

Central European University, Budapest - IREMAM-AMU

\section{Résumé}

Les gauches radicales au Liban naissent au cours des années 1960-1970 des processus de radicalisation et de marxisation des courants panarabes. Elles adoptent une posture critique à l'égard de l'URSS, des PC arabes et du parti communiste libanais et s'inscrivent dans des dynamiques contestataires transnationales. Pour dégager les jeux de tensions et de convergences entre la formation d'un univers transnational orienté par des espérances révolutionnaires et la redéfinition des enjeux stratégiques, cet article propose d'analyser les modes de référence au Vietnam. Nous montrerons comment les références multiples à ce modèle lointain agissent aussi bien dans les recompositions de la scène militante que dans la reconfiguration du sens et de valeurs.

Mots-clefs: Histoire transnationale - gauches arabes - nouvelles gauches - révolution - violence.

\begin{abstract}
Modes of Reference to Vietnam and the Rise of Radical Left in Lebanon, 1962-1976

During the 1960s and 1970s, the Radical Left in Lebanon took shape through its critical stance towards the Lebanese Communist Party, the USSR, and pan-Arab movements, and was embedded in transnational protest networks. I propose to explore the modes of reference to Vietnam in order to decipher the interplay of tensions and convergences between the redefinition of strategic challenges and the reframing of a transnational universe of meaning, oriented by revolutionary hopes. I show how the multifarious references to this distant model are at play in both the transformations in the militant landscape and the reconfiguration of meanings and values.
\end{abstract}

Keywords: Transnational History - Arab Left - New Left - Revolution - Violence. 
$\mathrm{L}$ es «nouvelles gauches » (al-yassar al-jadid) au Liban se forgent dans l'expérience de la défaite et de la désorientation. Si l'arabisme, dans sa version nassérienne et dans une moindre mesure baathiste, reste le courant anti-impérialiste dominant au cours des années 1950, l'échec de la République arabe unie (RAU, 1958-1961) indique dès 1961 l'essoufflement du projet politique d'unité arabe. La défaite arabe face à Israël (1967) conforte une partie de la génération militante active dans les années 1940-1950 dans le sentiment de désillusion à l'égard des régimes arabes, si ce n'est de tout projet d'émancipation politique et sociale. En revanche, conjuguée à la multiplication des interventions américaines dans le monde, elle précipite les processus de radicalisation d'une génération militante plus jeune qu'anime un sentiment d'urgence révolutionnaire. En réponse au désarroi provoqué par les échecs successifs des stratégies régionales dans les années 1960, ces « orphelins ${ }^{1} »$ des courants panarabes ou des partis communistes se tournent vers les modèles révolutionnaires chinois, vietnamien, algérien, et cubain, mais aussi vers les marges dissidentes du parti communiste italien (PCI) ou le Mouvement des droits civiques aux ÉtatsUnis. Ils suivent avec passion les mobilisations

1 Ahmad Beydoun, «Liban socialiste. Conditions d'émergence et cheminement d'un groupe de jeunes militants "gauchistes" dans le Liban des années 1960 ", communication au colloque Générations arabes, Fondation Hugot du Collège de France et Fondation Moulay Hicham, Paris, juin 2012. Texte non publié, communiqué par l'auteur. ouvrières et la contestation étudiante de la France de 1968, ainsi que l'émergence de la New Left dans le monde anglophone. Certains d'entre eux participent à ces mouvements contestataires en Europe et, au fil des circulations militantes ou des parcours académiques, tissent des liens variés et plus ou moins étroits avec des militants, des organisations, et des partis de gauche en dehors du monde arabe. Au Liban, comme en Europe et aux États-Unis,

«La coupure, qui sépare cette Nouvelle Gauche de la gauche tout court, se fait sur les grands enjeux du moment [...]. L'orientation cognitive nouvelle que les intellectuels de la Nouvelle Gauche opposent aux partisans de la gauche traditionnelle s'organise autour d'une nouvelle interprétation de la théorie marxiste, d'une autre vision de la société socialiste à venir, d'une perspective stratégique et organisationnelle renouvelée et, enfin, de l'identification de nouveaux acteurs du changement ${ }^{2}$.»

Longtemps, les études arabes sont pourtant restées en retrait du champ de recherche sur les Global Sixties alors que, depuis la fin des années 1990, la volonté de mieux saisir les dynamiques historiques qui firent des années 1960 un « moment critique global ${ }^{3}$ » s'était accompagnée d'un renouvellement des

2 Ingrid Gilcher-Holtey, " La contribution des intellectuels de la Nouvelle Gauche à la définition du sens de Mai 68 ", in Geneviève Dreyfus-Armand, Robert Frank, Marie-Françoise Lévy, Michelle Zancarini-Fournel (dir.), Les années 68. Le temps de la contestation, Paris, Éditions Complexe, 2000, p. 89-90.

3 Jeremi Suri, Power and Protest. Global Revolution and the Rise of Detente (Harvard: Harvard University Press, 2003). 
approches et des questions. La recherche sur les mouvements contestataires, les espérances révolutionnaires et les transformations des subjectivités politiques avait porté l'attention sur les circulations militantes transnationales et élargi le champ d'observation aux pays du tiers-monde ${ }^{4}$. Stimulés par l'état de l'art et de l'historiographie aussi bien que par le désir de mieux comprendre les transformations sociales et politiques actuelles, les spécialistes du monde arabe se tournent à nouveau vers ces courants négligés par la recherche et resituent leur émergence dans une histoire mondiale des gauches ${ }^{5}$. Cependant, dans les études arabes,

4 Boris Gobille, "Introduction. Circulations révolutionnaires. Une histoire connectée et "à parts égales" des "années 1968" ", Monde(s). Histoire, espaces, relations, $\mathrm{n}^{\circ}$ 11, 2017, p. 13-36; American Historical Review Forum, "The International 1968", American Historical Review (2009/2), p. 42-44; American Historical Review (2009/3), p. 329-330; Samantha Christiansen, Zachary A. Scarlett, eds., The Third World in the Global 1960s (New York: Berghahn Books, 2013); Alberto Martin Alvarez, Eduardo Rey Tristan, eds., Revolutionary Violence and the New Left Transnational Perspectives (London: Routledge, 2017); Carol Fink, Philipp Gassert, Detlef Junke, eds., 1968: The World Transformed (New York: Cambridge University Press, 1998).

5 Fadi Bardawil, When All this Revolution Melts into Air: The Disenchantment of Levantine Marxist Intellectuals, Ph.D. Columbia University (New York, Columbia University, 2010); "Special Section: The Arab Left in Egypt and Lebanon", Arab Studies Journal (2016/1), p. 90-228; Abdel Razzaq Takriti, Monsoon Revolution: Republicans, Sultans, and Empires in Oman, 19651976 (Oxford: Oxford University Press, 2013); Pamela Pennock, The Rise of the Arab American Left: Activists, Allies, and Their Fight against Imperialism and Racism, 1960s-1980s (Chapel Hill: University of North Carolina Press, 2017). l'écart persiste entre une histoire diplomatique qui tend à subordonner les transformations symboliques et discursives aux enjeux stratégiques ${ }^{6}$, et une histoire intellectuelle et une anthropologie politique qui privilégient l'étude des trajectoires intellectuelles et militantes individuelles ${ }^{7}$.

Dans un premier temps, l'attention sera portée sur le Vietnam comme modèle révolutionnaire de guerre du peuple. Par-delà les circulations militantes, somme toute limitées, entre les aires arabe et Sud asiatique, et les expressions de solidarité qui restent souvent verbales, le Vietnam devient en effet une figure axiale de la «mise en sens ${ }^{8}$ » du réel. Aussi étudiera-t-on, dans un second temps, comment, en tant que modèle militaire incarnant l'espoir de la victoire, le Vietnam apparaît également comme un exemple politique, social, et éthique. Enfin, comme cas d'étude, il ouvre à la critique des régimes et des sociétés arabes.

Cet article repose sur l'analyse de sources d'époque et de textes contemporains, et sur des témoignages oraux recueillis au cours d'entretiens entre 2015 et 2017. Différents types de textes publiés par les figures

6 Paul Chamberlin, "The Struggle Against Oppression Everywhere: The Global Politics of Palestinian Liberation", Middle Eastern Studies (2011/1), p. 25-41.

7 Fadi Bardawil, "Dreams of a Dual Birth: Socialist Lebanon's World and Ours", Boundary 2 (2016/3), p. 313-335.

8 Claude Lefort, Essai sur le politique : $X I X^{e}-X X^{e}$ siècles, Paris, Le Seuil, 1986. 
majeures de la gauche radicale des années 1960-1970 ont également été consultés au Liban, depuis les écrits personnels jusqu'aux documents politiques de circonstance et aux investigations théoriques. La presse militante forme une partie importante de ce corpus, en particulier l'hebdomadaire Al-hurriya et la publication Lubnan al-Ishtiraki (Liban socialiste). Revue ronéotypée imprimée et distribuée clandestinement par l'organisation éponyme, Lubnan al-Ishtiraki reste un périodique confidentiel, à la mesure d'un groupe limité à quelques dizaines de militants. Elle compte 17 numéros et quelques études annexes parus de manière irrégulière entre 1966 et 1970 . Son intérêt réside dans la qualité des textes, écrits par de jeunes militants qui ont ensuite occupé des postes de direction dans l'Organisation de l'action communiste au Liban (OACL) et à la rédaction d'Al-hurriya. $\mathrm{Au}$ début des années 1960, Al-hurriya est initialement l'organe de la branche libanaise du Mouvement nationaliste arabe (MNA), puis est conjointement publié par le Front populaire démocratique de libération de la Palestine (FPDLP) - issu du MNA en 1969 - et l'OACL (1971-), la principale organisation de la Nouvelle Gauche au Liban, qui rassemblait plusieurs milliers de militants et constitua le creuset des élites intellectuelles actuelles.

\section{Le Vietnam comme modèle révolutionnaire}

Au début des années 1960, de nouvelles géographies de résistance se dessinent, et la référence à la lutte vietnamienne se trouve au cœur d'une reconfiguration des rapports entre local, régional, et global. Si les événements des années 1967-1968 accélèrent l'inscription des espérances révolutionnaires arabes dans un univers contestataire transnational, les gauches radicales scellent cependant le sort du projet politique régional panarabe et réorientent leurs actions aux échelles nationales et locales.

Créer des foci ou établir des Hanoï : plus ou moins confusément associée au maoïsme, la référence à la stratégie militaire vietnamienne est omniprésente dans les logiques de distinction infra- et inter-organisationnelles. Les revues militantes et culturelles publient des dizaines de textes sur la « guerre du peuple » qui portent sur la stratégie militaire et sur la théorie de la lutte révolutionnaire dans une perspective marxiste $^{9}$. En référence au combat vietna-

9 Par exemple : Al-'Afif al-Akhdar, « Manzurat al-harb alvitnamiya "[Conceptions vietnamiennes de la guerre], Dirasat 'arabiyya, $\mathrm{n}^{\circ} 7$, mai 1967, p. 17-55; Mustapha Talas, " Dirasat fi harb al-tahrir al-sha'biyya. Nushu wa takawun al-nazariyya al-shuyu'iyya fi harb al-'isabat " [Études sur la guerre de libération populaire. Genèse et développement de la théorie communiste de la guérilla], Dirasat 'arabiyya, $\mathrm{n}^{\circ}$ 7, mai 1967; Khalil Ahmed Khalil, « Durus thawria li al-vitnam wa al-sin » [Leçons révolutionnaires du Vietnam et de la Chine], Dirasat ‘arabiyya, n 8 , juin 1968, p. 86-99. 
mien et aux écrits de Mao Ze Dong, la notion de « guerre du peuple » joue un rôle central dans les jeux d'alliances sur les scènes régionale et internationale, mais également dans la redéfinition de l'ordre des priorités entre émancipation nationale (arabe) et révolution sociale. En effet, deux questions déterminent la clôture des débats aussi bien que les alliances stratégiques dans les espaces politiques arabes depuis la création de l'État d'Israël en 1948 : le sort de la Palestine et la relation entre libération nationale (arabe) et socialisme. Jusqu'à la fin des années 1950, les mouvements panarabes s'accordent en général à poser l'unité arabe comme condition préalable à la libération de la Palestine et, partant, sur la priorité de l'étape politique par rapport à l'étape sociale-économique, bien que les positions divergent au sujet de l'épineuse question du leadership et des modalités de réalisation de ces objectifs. Au cours des années 1960, un nombre croissant de nationalistes arabes se réclame du marxisme-léninisme, accélérant les scissions et les dissensions au sein du Baath comme $\mathrm{du}$ Mouvement nationaliste arabe (MNA), dont l'origine remonte à la fin des années 1940 dans l'entourage de Georges Habash (Lod, 1926- Amman, 2008), alors étudiant en médecine à l'université américaine de Beyrouth.

\section{La « guerre du peuple » et la recomposition de la scène militante}

L'appel à la « guerre du peuple » représente le plus petit dénominateur commun aux gauches radicales libanaises et palestiniennes qui émergent au cours des années 1960 puis cristallisent à partir de 1967. Jusqu'à la fin des années 1960, l'appel à la lutte armée populaire constitue également l'un des points de rupture entre les nouvelles gauches et le parti communiste libanais (PCL) ou les organisations panarabes. Du PCL, du parti arabe Baath et du Mouvement nationaliste arabe (MNA) naissent, respectivement, l'Union des communistes libanais (1968-1970), Liban socialiste (1964-1971), et l'Organisation des socialistes libanais (1969-1971), qui fusionnent en 1971 dans l'Organisation pour l'action communiste au Liban (OACL). Présentons brièvement ces organisations.

Discrédités pour avoir accepté le plan onusien de partition de la Palestine en 1947, les PC arabes ont généralement continué à s'aligner de gré ou de force sur la ligne politique de Moscou et des régimes arabes dits progressistes qui, en Syrie et, en premier lieu, en Égypte, avaient monopolisé l'énonciation du discours de libération. Entrant en confrontation avec une vieille garde qui reste souvent proche de la politique prosoviétique du leader du parti communiste syrien - Khaled Bakdash (1912-1995) -, de jeunes cadres 
du PCL, tels Georges Hawi et Karim Mroué, entreprennent de réformer l'appareil de l'intérieur et parviennent à des positions de direction au sein du parti. Par la suite, lors de son deuxième congrès (1968), le PCL amende sa position sur la question de Palestine et marque une indépendance relative par rapport à $\mathrm{Moscou}^{10}$. En revanche, d'autres militants restent critiques à l'égard de la politique du PCL et de l'absence de démocratie interne au Parti, et ont opté pour la dissidence au milieu des années 1960 : en 1968, l'Union des communistes libanais (19681970) regroupe le " courant léniniste », issu du comité central du parti en 1964, et les " cercles marxistes-léninistes », formés par des étudiants des établissements français de la capitale en 1964-1965 sous influence guévariste aussi bien que trotskiste et maoïste.

Le noyau fondateur du groupe Liban socialiste, créé en 1964, est principalement constitué de jeunes nationalistes arabes déçus du baathisme, tels Fawwaz Traboulsi (1941-) et Waddah Charara (1942-), puis Ahmad Beydoun (1943-), qui rejoint l'organisation en 1966. Traboulsi a effectué une partie de ses études à Manchester puis à Londres, où

10 Tareq Y. Ismael, Jacqueline S. Ismaël, The Communist Movement in Syria and Lebanon (Gainsville: University Press of Florida, 1998); Karim Mroué, Un demi-siècle d'utopie, mémoires d'un dirigeant de la gauche libanaise, Beyrouth-Paris, Ifpo-Téraèdre, 2009; Al-hizb al-shuyu'i al-lubnani (PCL), Al-watha'iq al-kamila li almu'tamar al-watani al-thani li al-hizb al-shuyu'i al-lubnani [Les documents complets du deuxième congrès national du PCL], Beyrouth, Publications du PCL, 1988. il rencontre les principaux acteurs de la New Left, tandis que Beydoun et Charara étudient en France, où ce dernier entre notamment en contact avec la Gauche prolétarienne ${ }^{11}$. L'échec de l'union syro-égyptienne en 1961 constitue un traumatisme pour de nombreux membres ou sympathisants du Baath. Incriminant l'indigence théorique du nassérisme comme du baathisme, ils puisent dans de multiples traditions intellectuelles aussi bien que dans l'histoire contemporaine des mouvements de libération nationale et des révolutions pour s'engager dans une relecture du marxisme-léninisme, qui est également dirigée contre les PC arabes et l'URSS ${ }^{12}$. Ayant cessé d'attendre une solution de la part des régimes arabes, ils se tournent vers " les sociétés », conscients " de la nécessité de les connaître afin de les changer et, pour ce faire, de disposer d'une grille théorique

11 Pour une présentation de ces militants et de ce groupe : Ahmad Beydoun, "Liban socialiste ", op. cit. (cf. note 1); Fadi Bardawil, "Dreams", op. cit. (cf. note 7); Fadi Bardawil, "When these Revolutions", op. cit. (cf. note 5); Agnès Favier, "Logiques de l'engagement et modes de contestation au Liban : genèse et éclatement d'une génération de militants intellectuels (1958-1975) ", thèse de doctorat en sciences politiques, sous la direction de Yves Schemeil, université Aix-Marseille 3, 2004.

12 Lubnan Ishtiraki [Liban socialiste], « Madkhal li qira'at al-bayan al-shuyu'iłi » [Introduction à la lecture du Manifeste Communiste], Dirasat 'arabiyya, $\mathrm{n}^{\circ}$ 7, 1969, p. 38-80; Fadi Bardawil, "Dreams", op. cit. (cf. note 7); Laure Guirguis, "The Lebanese New Left and 1917 as Model and Foil", in Laura Feliu Martinez, Ferran Izquierdo, Francisco Veiga, eds., The Communist Parties in the Middle East: A History of a Century (London: Routledge, 2018). 
adéquate $^{13} »$, explique le sociologue et philosophe Ahmed Beydoun au cours d'une analyse rétrospective du cheminement de Liban socialiste. Après la défaite des armées conventionnelles des États arabes face à Israël, « en 1967, le concept qui véritablement s'impose, c'est celui de la lutte armée, et l'idée que la guerre doit être menée par le peuple. Les textes de Giap, Mao et Che étaient lus avec avidité et ont contribué à la radicalisation militante de l'après 67 », analyse Fawwaz Traboulsi ${ }^{14}$ (1941-), actuellement professeur d'histoire à l'université américaine de Beyrouth, membre du Baath et sympathisant du MNA au début des années 1960, puis fondateur de Liban socialiste (19641971) et numéro 2 de l'OACL (1971-...).

« Le Vietnam nous a enthousiasmés, se souvient Ahmad Beydoun. En revanche, nous éprouvions quelques réticences à l'égard du guévarisme, et de la théorie du foco, surtout après le départ du Che pour la Bolivie et la parution du texte de Debray. Forcément, nous dressions un parallèle entre ces deux conceptions de la lutte révolutionnaire, et, même si nous n'avons pas alors formulé de critique de la théorie du foco comme nous en avons formulé à l'égard du PCL, c'était, clairement, du côté du Vietnam que nous penchions, car il représentait pour nous la mise en œuvre de la guerre du peuple prônée par $\mathrm{Mao}^{15}$. »

13 Ahmad Beydoun, "Liban socialiste ", op. cit. (cf. note 1).

14 Entretien avec l'auteur, 13 novembre 2016, Beyrouth.

15 Entretien avec l'auteur, juin 2017, Beyrouth.
Du MNA, enfin, émergent plusieurs organisations. La question du mode de lutte constitue l'un des principaux clivages au sein du MNA et met directement en jeu la théorie des étapes, ainsi que le choix du sujet révolutionnaire, du leadership du mouvement, et des alliances régionales. Dès 1957, deux tendances rivales se dessinent au sein de la direction du MNA. Tandis que, en 1959, Nayef Hawatmeh, Mohammad Kichli et Mohsen Ibrahim affirment que la structure socio-économique constitue le principal facteur déterminant l'évolution des sociétés arabes, la direction maintient l'idée que la priorité actuelle demeure politique ${ }^{16}$. La divergence apparaît publiquement en 1960 lorsque Mohsen Ibrahim publie sans préavis un article dans lequel il remet en question la théorie des étapes ${ }^{17}$. Il dirige ensuite la critique vers le leadership du MNA qui, limité, selon lui, par ses présupposés et ses intérêts de classe, c'est-à-dire petit-bourgeois, n'a pas perçu l'importance des facteurs économiques et sociaux et, par conséquent, a échoué à libérer la région du sionisme et de l'impérialisme, tout autant qu'à mener une révolution sociale et démocratique ${ }^{18}$. Le coup sécessionniste syrien qui met de facto un terme à la RAU au mois de septembre 1961, a exacerbé simultanément les tensions entre

16 Sur le MNA : Walid Kazziha, Revolutionary Transformation in the Arab World: Habash and his Comrades from Nationalism to Marxism (London: Charles Knight, 1975).

17 Mohsen Ibrahim, Al-hurriya, 2 mai 1960.

18 Ibid., 6-13-20 janvier 1964. 
les partisans de la lutte populaire armée en Palestine - Georges Habash -, et les avocats de la ligne nassérienne - M. Ibrahim. Le MNA entendait en effet servir de catalyseur au déclenchement d'une guerre conventionnelle entre les États arabes et Israël, sans entraîner prématurément Nasser dans une guerre qu'il n'aurait pas souhaitée. Le MNA change progressivement de position sous la pression de la branche palestinienne du MNA (Habash), concrétisée en 1964, et du Fatah qui, à partir de 1965, lance des expéditions armées contre Israël. Fondé en 1958 par Yasser Arafat et des militants palestiniens en exil dans les pays du Golfe après des études au Caire ou Beyrouth, le Fatah se présente comme un mouvement de libération nationale palestinien et prône la lutte populaire armée contre Israël. À partir de 1967, il situe le combat palestinien dans le cadre d'une lutte globale contre l'impérialisme et établit des contacts avec la République populaire de Chine (RPC). Plusieurs cadres du Fatah suivent des formations dans des camps d'entraînement en Chine et au Vietnam ${ }^{19}$. Jusqu'en 1967, le MNA

19 Yezid Sayigh, "Turning Defeat into Opportunity: The Palestinian Guerrillas after the June 1967 War", Middle East Journal (1992/2), p. 260; Abou lyad, Éric Rouleau, Palestiniens sans patrie : Entretiens avec Éric Rouleau, Paris, Fayolle, 1978; Fred Halliday, "Review of The Middle East in China's Foreign Policy 19491977 by Yitzhak Shichor", Arab Studies Quarterly (1983/1), p. 86-88; Jeremy Friedman, "Free at Last, Now What: The Soviet and Chinese Attempts to Offer a Road-map for the Post-Colonial World", Modern China Studies (2015/1), p. 259-292; Abdel Razzaq Takriti, Monsoon, op. cit. (cf. note 5). ne formule que des justifications mesurées de la lutte armée, dans le souci de maintenir l'équilibre entre conciliation avec la politique nassérienne qui dicte alors une " escalade contrôlée » et « conversion totale au mode de guérilla du Fatah ${ }^{20} »$.

Après la défaite des armées arabes en 1967, le MNA opère un ultime changement de cap. Dans le communiqué autocritique qui officialise ce revirement, il prévoit de transférer la direction du mouvement aux ouvriers et aux paysans, et se prononce en faveur d'une guerre populaire de libération de longue durée $^{21}$. Il coordonne des opérations avec le Fatah qui tente, durant une brève période, de mener le combat depuis l'intérieur des Territoires occupés ${ }^{22}$. Cependant, écartelé entre la référence à une lutte globale contre l'impérialisme et l'engagement accru sur le terrain palestinien tout en maintenant l'objectif d'une libération arabe, le MNA perd pied sur tous les fronts. Plusieurs branches régionales restent actives, au Koweït, dans le Sultanat d'Oman, où le mouvement avait préservé son autonomie, mais il disparaît en tant qu'organisation centralisée.

20 Yezid Sayigh, "Reconstructing Paradox: The Arab Nationalist Movement, Armed Struggle, and Palestine, 1951-1966", Middle East Journal (1991/4), p. 624.

21 Communiqué du MNA, Al-hurriya, $\mathrm{n}^{\circ} 377,4$ septembre 1967, p. $12-13 ; n^{\circ} 378,11$ septembre 1967 , p. 10-12; $n^{\circ} 379,18$ septembre 1967, p. 12-14.

22 Yezid Sayigh, "Turning Defeat", op. cit. (cf. note 19). 
Plusieurs démonstrations de force de la résistance palestinienne contribuent à justifier le choix de la guérilla et du feda'i comme sujet révolutionnaire. Au mois de mars 1968 à Karameh (Jordanie), ville frontalière devenue le QG du Fatah après 1967, quelques centaines de combattants palestiniens soutenus par l'armée jordanienne tiennent tête à plusieurs milliers de soldats de Tsahal $^{23}$. Bien que l'affrontement se solde par une victoire militaire israélienne, cette bataille mythifiée propulse le Fatah au premier plan et accélère les reconfigurations de la scène militante. Au mois de janvier 1969, M. Ibrahim crée l'Organisation des socialistes libanais (OSL), « ce nouveau parti marxisteléniniste révolutionnaire au $\operatorname{Liban}^{24}$ ». La faction de Habash (Front populaire de libération de la Palestine - FPLP) entérine la division du MNA au mois de mars $1969^{25}$. La même année, après un bref passage par le FPLP, Hawatmeh annonce la création du Front populaire démocratique de libération de la Palestine (FPDLP), qui se revendique

23 Benny Morris, Righteous Victims: A History of the Zionist-Arab Conflict, 1881-2001 (London: Vintage, 2001).

24 Mohsen Ibrahim, Limadha? Munazzama al-ishtirakiyyin al-lubnaniyyin. Haraka al-qawmiyyin al-'arab min alfashiya ila al-nasiriyya. Naqd wa tahlil [L'Organisation des socialistes libanais, pourquoi? Le Mouvement des nationalistes arabes, du fascisme au nassérisme. Critique et analyse], Beyrouth, dar al-tali‘a, 1970.

25 Walid Kazziha, Revolutionnary, op. cit., p. 101 (cf. note 16). également du marxisme-léninisme ${ }^{26}$. Aucun des deux Fronts de libération ne se transforme en organisation de masse, au contraire du Fatah qui parvient à politiser la lutte, prend la direction de l'Organisation de libération de la Palestine (OLP), et gagne une stature internationale. Principale représentante de la nouvelle gauche au Liban, l'Organisation de l'action communiste au Liban (OACL) naît en 1971 de la fusion entre Liban socialiste (1964) et l'OSL, après une période transitionnelle de travail en commun à la rédaction de l'hebdomadaire Al-hurriya (financé par le FPDLP).

\section{Guerre du peuple et redéfinition du sujet, de l'organisation et des alliances révolutionnaires}

Les divergences au sujet de la stratégie à adopter et des modes d'organisation de la lutte séparent les nouvelles organisations des anciennes. Elles opposent également ces nouveaux groupes entre eux et parfois les scindent de l'intérieur. Les lignes de fractures entre et au sein de ces mouvements changent entre 1967 et 1973 (guerre du Kippour), puis en 1975 (début des guerres libanaises). Analysant point par point le communiqué autocritique publié par le MNA en 1967, l'un des auteurs anonymes de Liban socialiste

26 « Ta'sis al-jabha al-sha'biyya al-dimuqratiya li tahrir falistin " [Fondation du front populaire démocratique de libération de la Palestine], Al-hurriya, $\mathrm{n}^{\circ}$ 452, 24 février 1969; Communiqué du FPDLP, Al-hurriya, $\mathrm{n}^{\circ} 498$, 19 janvier 1970. 
pointe la faiblesse théorique et le manque de discernement du MNA, puis l'inadéquation des propositions stratégiques qui en résulte :

« La lutte armée est le sujet prioritaire du moment pour les mouvements de libération nationaux et sociaux dans le Tiers-monde. Sans aucun doute, la question s'impose au mouvement de libération arabe, non seulement à titre de partie du mouvement de libération des peuples d'Asie, d'Afrique, et d'Amérique latine, mais elle se pose à lui plus particulièrement car il passe actuellement par deux expériences de ce type de lutte : la révolution nationaliste au Yémen du sud et dans la péninsule arabique, et l'action feda'i palestinienne ${ }^{27}$. »

Or, poursuit l'auteur, faute d'une analyse rigoureuse - marxiste-léniniste - des spécificités de ces deux configurations géostratégiques, le MNA établit des analogies erronées entre les situations yéménite et palestinienne et dresse, par conséquent, des plans d'action voués à l'échec. De plus, il ne tire pas de la défaite de 1967 les conclusions qui s'imposent : il ne s'agit pas simplement de la faillite des régimes arabes, mais de l'échec d'une culture politique, de la politique par coup d'État. S'il se tourne finalement vers le peuple en préconisant de transférer la direction du Mouvement aux ouvriers, aux paysans et à certaines catégories de la petite

27 Lubnan Ishtiraki, « Maza ya'ni raf' shira' al-kifah al-maslaha fi al-marahla al-rahina? " [Que signifie lancer l'appel à la lutte armée dans la situation actuelle?], Lubnan Ishtiraki, $\mathrm{n}^{\circ} 8$, décembre 1967. Sur la pratique de l'anonymat dans Liban socialiste : Fadi Bardawil, "Dreams", op. cit. (cf. note 7). bourgeoisie, il n'engage aucune action en ce sens, et ne définit pas clairement les contours de ces catégories de la petite bourgeoisie. Enfin, l'appel incantatoire au peuple ignore le fait que toute action guidée par la seule « spontanéité populaire désorganisée » est vouée à l'échec. En somme, l'appel du MNA à la guerre du peuple ne fixe pas l'objectif principal à l'étape actuelle : la formation d'un parti marxiste-léniniste qui ne se contente pas d'accompagner un mouvement spontané, mais qui forme et encadre le sujet révolutionnaire - les paysans, les ouvriers, et certaines catégories de la petite bourgeoisie ${ }^{28}$.

Au sein même de l'OACL, dès l'étape préparatoire de l'union entre Liban socialiste et l'Organisation des socialistes libanais, les références vietnamiennes et maoïstes creusent la divergence entre plusieurs conceptions des rapports classe/parti et base/parti, des alliances, et des conditions de transformation sociale. D’autant plus que l'évolution rapide de la situation libanaise désoriente les militants de Liban socialiste, jusqu'alors non encore confrontés à la violence endémique. Leurs activités consistaient pour une part importante en un travail de relecture critique, d'enseignement, et de discussion du corpus marxiste-léniniste. Or, dès 1968, les habitants des villages du sud développent une hostilité croissante à l'égard des Palestiniens dont la présence attire les représailles israéliennes, tandis que la

28 Lubnan Ishtiraki, id. 
répression des combattants palestiniens par la monarchie hachémite jordanienne au mois de septembre 1970, "Septembre noir », force un nombre grandissant de combattants à l'exil au Liban. À partir d'une relecture de Lénine (en particulier d'État et révolution), Traboulsi et Ibrahim conçoivent une démocratie centralisée, structurée, certes, par une hiérarchie pyramidale, mais qui prévoit des instances médiatrices et des moments de concertation permettant la constitution dialectique de la base/classe et du parti ${ }^{29}$ - la pratique s'éloigne de la théorie : le groupe indépendant de l'OACL (Beydoun et Charara) et plusieurs militants reprochent au leadership son exercice secret et autoritaire du pouvoir $^{30}$. Ahmad Beydoun et Waddah Charara défendent l'idée d'une démocratie cellulaire qui privilégie les liens horizontaux entre les cellules et leurs membres, et qui se démarque de la rigidité hiérarchique caractéristique du PCL. Corrélativement, en 1972-1973, Charara définit la « ligne de masses » comme une confrontation entre les masses et leurs ennemis qui se produit dans tous les secteurs sociaux et toutes les positions de pouvoir,

29 Ahmad Beydoun, Waddah Charara, Al Majmu'a al mustaqilla (al-munazzama al 'amal al-shuyu'i) [Le groupe indépendant de l'Organisation de l'action communiste au Liban], Beyrouth, s.d. (ronéotypé); Ahmad Beydoun, "Liban socialiste ", op. cit. (cf. note 1); Fawwaz Traboulsi, Sura al-fatat bil-lun al-ahmar [Portrait du jeune homme en rouge], Beyrouth, Riad El-Rayyes Books, 1997, p. 115-120.

30 Ahmad Beydoun, Waddah Charara, id.; Dalal al-Bizri, Sanawat al-sa'ada al-thawriya [Les années de joie révolutionnaire], Beyrouth, Dar al-tanwir, 2016. et la guerre du peuple comme l'irruption de la violence des masses sur ces lignes de confrontation, sans l'intermédiaire d'une avant-garde éclairée ${ }^{31}$. Dans une perspective différente, la même notion sera utilisée par la gauche du Fatah de tendance maoïste, dont Mounir Chafiq est la figure la plus emblématique. Palestinien chrétien exilé au Liban, Chafiq participe en 1973 à la création des brigades étudiantes du Fatah qui, initialement d'orientation maoïste, se définissent à partir de la fin des années 1970 par leur ancrage dans l'islamité, tout en considérant ce tournant idéologique comme une réponse à une préoccupation unique, celle de toucher les masses $^{32}$.

La question de l'organisation se noue à celle du choix des alliances aux échelles locales, régionales, et mondiales.

" La lutte vietnamienne représentait l'un des motifs de critique de l'URSS. Liban socialiste ne se faisait pas d'illusion au sujet de l'URSS, dont on dénonçait le manque d'empressement à soutenir les Vietnamiens, puis les armées arabes durant la guerre de 1967. L'antisoviétisme de Liban socialiste était très prégnant et n'occupait pas moins de place dans les imaginaires militants que l'antiaméricanisme ${ }^{33}$ ",

31 Ahmad Beydoun, Waddah Charara, ibid., p. 91.

32 Nicolas Dot-Pouillard, « De Pékin à Téhéran, en regardant vers Jérusalem : la singulière conversion à l'islamisme des "Maos du Fatah" ", Cahier de I'Institut Religioscope, 2008/2; Manfred Sing, "Brothers in Arms: How Palestinian Maoists Turned Jihadists", Die Welt des Islams, 2011/1, p. 1-44.

33 Entretien avec l'auteur, 9 mai 2017, Amsterdam-Beyrouth. 
se souvient Ahmad Beydoun. En dépit des conséquences désastreuses du Grand Bond en avant, longtemps méconnues hors de Chine, la RPC apparaît comme un modèle de développement alternatif et comme un allié possible. Pourtant, si la Chine, qui avait soutenu le FLN algérien, accorde une aide logistique au Front populaire de libération du Golfe arabique occupé (FPLGAO) et au Fatah, ce soutien demeure bref et limité. Certes, dans les années 1960, la traduction et la diffusion des textes de Mao Ze Dong représentent un axe fondamental de la politique tiers-mondiste de la $\mathrm{RPC}^{34}$. Mais cet effort s'essouffle dès la fin des années 1960, tandis que le prestige du maoïsme grandit. En d'autres termes, l'impact du maoïsme et du modèle vietnamien sur la transformation des imaginaires et des ethos militants arabes apparaît partiellement désarticulé de la politique arabe de la Chine ${ }^{35}$. En 1971, toutefois, la position du Parti communiste chinois en faveur de Numeiri, qui ordonne le massacre des communistes soudanais soupçonnés d'avoir participé à la tentative de coup d'État,

34 Cheng Yinghong "Xiang shijie shuchu geming : wege zai Yafeilayingxiang de chutan "[Exporter la révolution : étude préliminaire sur l'influence de la révolution culturelle sur les régions d'Asie, d'Afrique et d'Amérique latine], in Mao zhuyi yu shijie geming [La révolution maoïste et le monde au XXe siècle], Hong Kong, Tianyuanshuwu, 2009, p. 59-100.

35 Jeremy Friedman, "Free at Last, Now What: The Soviet and Chinese Attempts to Offer a Road-map for the Post-Colonial World", Modern China Studies (2015/1), p. 259-293. modère les espoirs placés dans la Chine maoïste.

Sur la scène régionale et au Liban, Traboulsi et Ibrahim restent proches du FPDLP et optent résolument pour l'alliance avec le Front des partis, des forces et des personnalités progressistes qui rassemble tant bien que mal le PCL, le parti socialiste progressiste (PSP) et diverses personnalités indépendantes sous l'égide du leader druze et chef du PSP, Kamal Joumblatt, et qui, en 1975, se renomme Mouvement national libanais ${ }^{36}$. Or, Liban socialiste avait préalablement refusé l'alliance avec les forces considérées comme représentantes de la bourgeoisie nationale. Pour leur part, Beydoun et Charara entendent

« nouer des relations directes et à la base avec des organisations palestiniennes et, dans cette perspective, avec le Fatah, qui est une organisation de masse, plutôt qu'avec le FPDLP, avec lequel il y avait déjà eu des différends dans le cadre de la publication d'Al-hurriya, dont le marxisme est "un peu douteux", et qui entretient des relations étroites avec les régimes irakien et libyen »

explique Ahmad Beydoun ${ }^{37}$. «Au bout d'une année environ, cette faction, qui, enfin, avait opté ouvertement pour le maoïsme, avait cessé d'exister. L'autre tint bon et put

36 Lubnan Ishtiraki, $\mathrm{n}^{\circ}$ 9, février 1968; sur Kamal Joumblatt : Farid Al-Khazen, "Kamal Jumblatt, the Uncrowned Druze Prince of the Left", Middle Eastern Studies (1988/2), p. 178-205.

37 Entretien avec l'auteur, 27 octobre 2016, Beyrouth. 
s'enfoncer dans la guerre civile ${ }^{38}$. " Beaucoup poursuivent le combat jusqu'à l'invasion israélienne de 1982 et après, même si la plupart d'entre eux ne conçoivent plus, à cette période, la résistance palestinienne comme le fer de lance d'une révolution de la société libanaise; certains affirment aujourd'hui ne l'avoir jamais crue et avoir toujours considéré le soutien à la résistance palestinienne comme une lutte défensive ${ }^{39}$.

Le Fatah place la question palestinienne - et non pas arabe - au cœur de sa stratégie tout en la situant dans le cadre d'une guerre globale contre l'impérialisme. L'appel à la guerre $\mathrm{du}$ peuple constitue un élément décisif du processus de construction d'une identité nationale palestinienne, au moment même où la résistance s'organise principalement depuis divers sites d'exil, en Jordanie, puis au Liban après « Septembre noir ${ }^{40}$ ». Opérant dans un mouvement similaire, les militants $\mathrm{du}$ groupe Liban socialiste, puis de l'OACL, découvrent les spécificités de la « situation libanaise » que la focalisation sur les enjeux arabes avait auparavant écartées de leurs préoccupations prioritaires. Et, dès 1969,

38 Ahmad Beydoun, "Liban socialiste ", op. cit. (cf. note 1); Sur la scission de l'OACL, voir aussi : Fawwaz Traboulsi, Sura, op. cit. (cf. note 29); « Bayan min almaktab al-siyasi al-munazzama al-'amal al-shuyu'i fi lubnan " [Communiqué du bureau politique de l'organisation de l'action communiste au Liban], Al-hurriya, $n^{\circ}$ 628, 16 juillet 1973, p. 12-15.

39 Entretien avec Nahla Chahal (PCL jusqu'en 1969, puis OACL), 27 mai 2017, Beyrouth.

40 Yezid Sayigh, “Turning Defeat”, op. cit. (cf. note 19).
Charara pointe la nécessité de distinguer deux résistances, libanaise et palestinienne, et défend l'idée d'une alliance qui préserve l'autonomie de chaque partie ${ }^{41}$.

\section{Violence révolutionnaire et exigence démocratique}

Le Vietnam acquiert une valeur paradigmatique non seulement à titre de modèle de lutte révolutionnaire et d'organisation sociale et politique mais dans la mesure où il redonne sens et espérance au cours d'une époque de désorientation.

Aux États-Unis, le refus du départ des citoyens américains pour cette guerre lointaine constitue un facteur décisif de mobilisation en faveur de la paix ${ }^{42}$. L'image de la résistance de David face à Goliath suscite l'enthousiasme des militants arabes :

« Le combat que mène le peuple vietnamien contre la férocité des forces impérialistes à notre époque est un combat inexorable : le peuple vietnamien ne peut que vaincre ou être anéanti. [...] L'avenir des peuples dépend pour une grande part de cette victoire. Si le peuple vietnamien perd cette guerre, il perd tout : son indépendance, sa liberté, sa joie, et le riz qu'il cultive, il perd sa raison d'être. Aussi défend-il héroïquement sa terre. Mais il défend en même

41 Waddah Charara, «Al-muqawamatayn » [Les deux résistances], Lubnan al-Ishtiraki, n 16, n 17, décembre 1969.

42 Geneviève Dreyfus-Armand, Jacques Portes, "Les interactions internationales du Viêt-nam et Mai 68 ", in Geneviève Dreyfus-Armand et al., Les années 68, op. cit., p 51-52 (cf. note 2). 
temps l'ensemble des intérêts socialistes du monde, l'ensemble des forces révolutionnaires et émancipatrices et des peuples assujettis. Sa victoire est leur victoire et sa défaite leur défaite ${ }^{43}$.»

Ce texte n'expose pas simplement un point de vue stratégique. Aux analogies - « comme le peuple vietnamien vainc, le peuple palestinien vaincra ${ }^{44} »-$ se conjugue l'idée que l'issue du combat sur le front vietnamien engage le sort de la révolution mondiale. Symbole, le combat vietnamien cristallise une vision du monde, un monde polarisé sur les plans politiques, stratégiques, et idéologiques. Exemple de résistance héroïque, il apparaît comme la métonymie du tout et signifie de la sorte l'unité, jamais réalisée, de forces variées. Image du tout, le Vietnam indique également sa transformation, possible ou inéluctable. C'est une image-écran, elle offre un espace de projection aux espérances révolutionnaires qui mettent en jeu une configuration temporelle orientée vers l'avenir.

Les images - ou les noms propres - de ses deux figures emblématiques, Hô Chi Minh et Giap, entrent en résonance selon des modalités différenciées dans les imaginaires militants. Le second apparaît principalement comme un chef militaire, un grand stratège, et un théoricien de la lutte armée qui a su

43 Lubnan Ishtiraki, "Muhawulat fi fahm al-harb al-vitnamiya " [Essai d'analyse de la guerre du Vietnam], Lubnan Ishtiraki, $\mathrm{n}^{\circ} 11$, mai 1968, p. 16-33.

44 Photographie d'une pancarte en français lors d'une manifestation à Paris, Al-hurriya, 469, 23 juin 1969, p. 1. élaborer une conception originale par rapport à celle de Mao Ze Dong ${ }^{45}$. À l'instar du Che, Hô, " A'zam qa'id li a'zam thawra didd ilisti'mar ${ }^{46}$ ", est tour à tour symbole et figure allégorique. Le slogan « Hô, Hô, Hô Chi Minh, Che, Che Guevara [... qui] scandé à Berlin puis à Paris liait [...] ces deux hommes dans le même rythme protestataire ${ }^{47}$ », résonne aussi sur les trottoirs de Beyrouth, dans les manifestations de l'OACL, comme dans les rangs du Fatah ${ }^{48}$. « Nous étions jeunes, nous étions naïfs, nous voulions transformer le Liban en un Hanoï, mais nous avons été écrasés par des forces plus puissantes que nous ${ }^{49}$ ", témoigne l'universitaire libanais Sa'ud alMaula, membre de l'Organisation de l'action communiste au Liban (OACL) puis du Fatah. Modèle militaire et symbole du courage, Hô représente aux yeux de nombreux militants la violence révolutionnaire mais aussi la paix et la démocratie. Figure allégorique, il

45 Mustapha Talas, « Dirasat fi harb al-tahrir al-sha'biyya », op. cit. (cf. note 9).

46 " Hô Chi Minh : A'zam qa'id li a'zam thawra didd ilisti'mar » [Hô Chi Minh : le plus grand leader de la plus grande révolution contre l'impérialisme], Al-hurriya, $n^{\circ} 480,8$ septembre 1969, p. 8.

47 Robert Frank, "Imaginaires politiques et figures symboliques internationales: Castro, Hô, Mao et le Che ", in Geneviève Dreyfus-Armand et al., Les années 68, op. cit., p. 32 (cf. note 2).

48 Dalal al-Bizri, Sanawat, op. cit. (cf. note 30); Fawwaz Traboulsi, Șura, op. cit. (cf. note 29).

49 Témoignage de Sa'ud al-Mauwla, My Hearts Beats only for Her, film réalisé par Mohammed Soueid. " Her » se réfère à la résistance palestinienne. 
incarne la tension entre violence révolutionnaire et exigence démocratique.

Le Vietnam apparaît en effet comme un modèle politique qui allie construction nationale et transition supposée réussie vers le socialisme. En ce sens, le Vietnam figure la conciliation entre ce qui, dans le monde arabe, se présente en général sous la forme d'une alternative, si ce n'est d'un antagonisme : révolution sociale-économique ou politique nationale, tradition supposée endogène ou modernité considérée comme importée. Dans un compte rendu de l'ouvrage de Gérard Chaliand sur la paysannerie du Nord Vietnam, le militant tunisien Al-Afif Al-Akhdar (un avocat proche de Ben Bella qui, en 1965, a migré vers Amman où il entre en contact avec le FPDLP avant de s'installer à Beyrouth jusqu'en 1979), voit dans la commune vietnamienne un exemple d'harmonie entre intégration des structures villageoises traditionnelles et modernisation-démocratie socialiste ${ }^{50}$.

Le combat met également en jeu une opposition de valeurs et de normes. La revue culturelle al-Tariq, proche du PCL, intitule l'éditorial de mars-avril 1966 « La cause de l'humanité au Vietnam ${ }^{51} »$. Quelques jours

50 Al-'Afif al-Akhdar, «Al-komiyun al-vitnami wa al-dimuqrati al-ishtiraki »[La commune vietnamienne et la démocratie socialiste], Al-hurriya, $\mathrm{n}^{\circ} 498,19$ janvier 1970, p. 10, 11, 15.

51 Éditorial, «Ma'a qadiya al-insan fi al-vitnam » [La cause de I'humanité au Vietnam], Al-tariq, 3-4, mars-avril 1966, p. 2-4. après la défaite de 1967, Mohammed Kichli, membre du MNA puis de l'OACL, lit dans

« la confrontation avec le colonialisme, non seulement, une confrontation entre deux armées mais entre deux volontés : la volonté d'exploiter et d'assujettir, la volonté tyrannique, sur laquelle repose la puissance militaire du capitalisme colonialiste, et la volonté de liberté des peuples combattants $^{52}$ ».

Commentant le texte de Chaliand, Al-Afif alAkhdar évoque une formule affichée à l'entrée des villages vietnamiens : "Ne tuez pas le temps, emplissez-le de vie ${ }^{53}$. " La télescopant avec l'expression « Le temps, c'est de l'argent », qu'il estime représentative de la Weltanschauung prédominante des bourgeoisies occidentales, il brosse le tableau de deux mondes de valeurs antagonistes et de deux conceptions inconciliables de l'existence et de la temporalité : l'une, capitaliste-impérialiste, définie à l'aune de l'argent, et l'autre, socialiste-tiers-mondiste, à l'aune de la vie ${ }^{54}$.

Paradigme, le Vietnam recèle une valeur mobilisatrice ${ }^{55}$, et heuristique :

"Lorsque nous écrivons sur le Vietnam, nous écrivons sur un exemple à suivre et sur l'idéal

52 Mohammad Kichli, « Haza huwa al-shi'ar : harb kamila ma'a amirika » [Le mot d'ordre c'est : guerre totale contre l'Amérique], Al-hurriya, n³66, 19 juin 1967, p. 5.

53 Al-'Afif al-Akhdar, «Al-komiyun al-vitnami », op. cit. (cf. note 50 ).

54 ld.

55 Par exemple, le discours de Georges Habash, Al-hadaf, 24 mars 1973. 
vers lequel nous tendons. L'écriture est l'image mais la lutte c'est l'action qui assure le renouvellement de la vie, actualise ses potentialités, lui confère sa richesse. Nos écrits sur le Vietnam ne sont que l'écho de cet hymne épique, nationaliste et internationaliste, nos paroles ne sont que l'image de l'action sanglante, mais nous devons écrire, car il faut que notre peuple voie cette image, notre peuple opprimé qui ne sait pas, le plus souvent, pourquoi il est broyé, ni comment se relever de son affaissement ${ }^{56}$. »

\section{(Auto-)critiques arabes : le Vietnam comme contre-exemple}

Allégorie ou symbole de la victoire et d'un monde nouveau, le Vietnam figure en creux la réalité de la défaite. Plusieurs penseurs et militants de gauche ont formulé des critiques a contrario des politiques arabes à partir d'une analyse comparée des situations vietnamienne et arabe. Bien qu'ils répondent à des préoccupations politiques et théoriques différentes, Samir Frangie et Yasin al-Hafez engagent tous deux la réflexion à partir de l'interrogation, maintes fois ressassée, sur les raisons de la victoire vietnamienne : comment un pays qui ne disposait pas initialement de davantage d'atouts que les pays arabes a-t-il tenu tête à la plus grande puissance militaire et politique du monde? Frangie pointe l'échec de la résistance

56 Al-'Afif al-Akhdar, "Al-komiyun al-vitnami », op. cit. (cf. note 50); Gérard Chaliand, Les paysans du NordVietnam et la guerre, Paris, Éditons Maspero, 1968. palestinienne à initier une révolution socialiste, tandis que Yasin al-Hafez entreprend une critique historique de la raison arabe.

Samir Frangie (1945-2017) est issu d'une famille influente de Zghorta qui donna naissance à plusieurs hommes politiques de premier plan, dont son oncle, Soleiman Frangie, président du Liban de 1970 à 1976, proche du clan Assad et membre de la coalition de l'extrême droite chrétienne, le Front libanais, jusqu'en 1978. Samir, en revanche, le Bey rouge, actif dans les organisations étudiantes de gauche des années 1960-1970, passe brièvement par l'OACL et rejoint le Mouvement National Libanais en 1975. La brutalité des premières années de guerre, ponctuées par divers massacres mais aussi par l'assassinat de Kamal Joumblatt et de Tony Frangie, lui ôte l'illusion que la résistance palestinienne puisse atteindre ses objectifs par la violence. Dès 1978, il s'engage dans diverses initiatives pour tenter de rapprocher les belligérants. Également chroniqueur dans le quotidien L'Orient-Le Jour, il est l'auteur de plusieurs essais politiques. Quelques mois après Septembre noir (1970), il estime que les analyses des erreurs de la résistance palestinienne n'identifient pas la racine $d u$ problème ${ }^{57}$.

Principalement formée d'une population de réfugiés « dominée par l'idée d'un retour en

57 Ghayth Armanazi, "September Post-Mortems", Journal of Palestine Studies (1974/2), p. 130-136. 
Palestine $^{58} »$, la résistance palestinienne n'est pas intégrée dans le processus de production économique non plus que dans le tissu social des pays d'accueil. Dans ces conditions, poursuit Frangie, la guerre du peuple se réduit purement et simplement à une lutte armée. Or, en Chine et au Vietnam, la guerre du peuple signifie une transformation des relations sociales et des rapports de production, dont la lutte armée ne représente qu'une partie. «Dans cette perspective, le politique a priorité sur le militaire qui n'est que l'un des moyens du changement ${ }^{59}$. " N'ayant aucune raison de modifier les structures sociales et économiques des régions d'accueil, les réfugiés-combattants palestiniens non seulement ont perdu le soutien des populations locales et sont devenus incapables de les mobiliser, mais ils se sont attiré leur hostilité. Dans le Sud, les habitants de Bint Jbeil, qui souffraient des exactions d'un " gouvernement allié au féodalisme local et du monopole du tabac », ont initialement exprimé leur solidarité avec les feda'in :

« Ils sentaient, confusément, qu'avec l'arrivée des commandos dans la région, "les choses allaient changer". Mais en fait rien n'a changé. Les feda'in, pour les raisons précédemment mentionnées, ne pouvaient pas joindre leurs forces à celles des populations locales pour transformer l'ordre social. Au contraire, ils ont

58 Samir Franjieh, "How Revolutionary is the Palestinian Resistance? A Marxist Interpretation", Journal of Palestine Studies (1972/2), p. 52-60.

59 lbid., p. 53. limité leur action aux opérations armées contre Israël, opérations qui ont provoqué des représailles ennemies dans les villages situés le long de la frontière ${ }^{60}$. »

Pour le penseur syrien Yasin al-Hafez, l'analyse comparée des expériences arabe et vietnamienne se situe dans le prolongement d'une lecture critique du marxisme orientée par les problèmes arabes ${ }^{61}$. Ainsi, dans les écrits publiés au moment de l'union de l'Égypte et la Syrie (1958-1961), entendaitil « donner un contenu socialiste à la lutte unioniste » et « orienter la lutte socialiste dans une direction unioniste ${ }^{62} »$, tout en formulant une critique acerbe des traditionalismes, stalinien aussi bien que nationalistes. Membre du Baath syrien depuis la fin des années 1940, il avait brièvement rejoint le parti communiste syrien puis coopéré à nouveau avec le Baath au début des années 1960 et contribué à la rédaction du rapport final du $6^{\mathrm{e}}$ Congrès (1963) avant de fonder, en 1966, au Liban, le parti révolutionnaire des travailleurs arabes. À partir du cas d'étude vietnamien, il poursuit l'effort de reformulation du rapport entre culturel-idéologique et social-économique : le Vietnam est un pays

60 Ibid., p. 54

61 Yasin al-Hafez, Al-tajriba al-tarikhiyya al-vitnamiyya. Taqiim naqdi muqarin ma'a al-tajriba al-tarikhiyya al-'arabiyya [L'expérience historique vietnamienne. Évaluation critique en comparaison avec l'expérience historique arabe], Beyrouth, Dar al-tali'a, 1976.

62 Yasin al-Hafez, Hawla ba'd qadaya al-thawra al-'arabiyya [Autour de quelques questions sur la révolution arabe], Beyrouth, Dar al-tali'a, 1965, p. 7-8. 
économiquement moins développé que les pays arabes, pourtant le Vietnam a mené à bien une révolution sociale et politique, tandis que les Arabes accumulent échecs et défaites. Al-Hafez explique par conséquent le « retard » arabe par le « retard » culturelidéologique non seulement des élites mais des sociétés arabes et, a contrario, interprète la victoire vietnamienne à partir du postulat selon lequel ce qui est structurel, c'est l'idéologie et la culture « qui gouvernent le politique [...]. Or, de quelque manière qu'ils soient, en dernière instance, liés à l'économique, le culturel, le politique et l'idéologique décident de l'autonomie ${ }^{63} »$.

Corrélativement à cet enjeu théorique, alHafez engage une critique des sociétés, des régimes et des intelligentsias arabes, critique qui, sur les traces du philosophe marocain, Abdallah Laroui, le conduit à réévaluer la pertinence du marxisme pour répondre aux problèmes arabes actuels et à se placer dans une perspective historiciste, un « historicisme de la désillusion ${ }^{64}$ ». Hafez écrit en 1976, quand le Liban s'enfonce dans une guerre qui a paralysé les aspirations démocratiques de nombreux militants pour ne répondre qu'aux tactiques miliciennes ultra-localisées mais enchâssées dans des luttes de pouvoir régio-

63 Yasin al-Hafez, Al-tajriba, op. cit. p. 7 (cf. note 61).

64 Samer Frangie, "Historicism, Socialism and Liberalism after the Defeat: on the Political Thought of Yasin al-Hafez", Modern Intellectual History (2015/2), p. 325-352. nales et internationales. Hafez ne renonce pas à la pensée révolutionnaire ni au marxisme comme outil d'analyse et comme orientation politique. Il parvient au constat amer que les élites et les sociétés arabes se trouvent dans un état de retard (ta'akhur) tel qu'une transition vers le socialisme apparaît impossible. Son historicisme signifie l'abandon de l'illusion d'un progrès automatique ou naturel des sociétés et implique une "réhabilitation ${ }^{65}$ » du libéralisme politique considéré comme moment de transition vers le socialisme : « La pensée historiciste est consciente du fait que l'on ne peut établir le socialisme sur la base de concepts et de système traditionnels mais seulement sur une base libérale ${ }^{66}$. »

Al-Hafez effectue une analyse comparée de la conscience historique - "L'image des événements passés tels qu'un peuple se les remémore et les interprète, et la position qu'il adopte à leur égard ${ }^{67}$ »-, et des postures politiques arabes et vietnamiennes. Il met en regard l'expérience arabe caractérisée par l'hégémonie du jugement de valeur, et l'expérience vietnamienne qui est passée, de façon dialectique, du jugement de valeur au jugement de fait puis à un jugement de valeur instruit par les faits ${ }^{68}$. Parallèlement, il décrit une politique arabe acculée à l'alternative

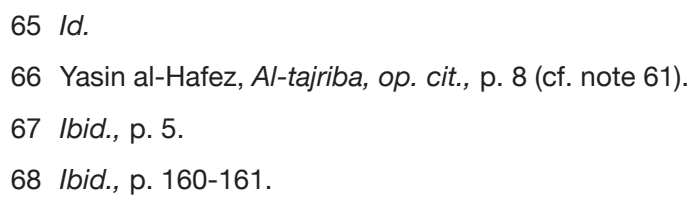


entre romantisme révolutionnaire et réalisme conservateur, se montrant particulièrement critique à l'égard de la troisième génération de l'intelligentsia arabe qui, étant « conservatrice sur les plans idéologiques, culturels, sociaux, était incapable de moderniser les structures sociales arabes, ce qui inclut l'économie [...]. Aussi son radicalisme politique a-t-il pris la forme du romantisme révolutionnaire ${ }^{69}$ "romantisme révolutionnaire que Hafez oppose au réalisme révolutionnaire vietnamien.

Alors que le Vietnam a su transformer les éléments de l'ancienne société, les Arabes ont reproduit des éléments des structures sociales et politiques traditionnelles dans le cadre de la société moderne : le confessionnalisme dans le contexte d'émergence du nationalisme (qawmiya), les rapports d'autorité ruraux reposant sur le clan et la confession dans la ville moderne, etc., opérant de ce fait une re-traditionalisation ${ }^{70}$. Les élites et la société vietnamiennes ont accompli une transition réussie vers le socialisme, car elles sont passées par le moment de libéralisme politique qui implique pluralisme et démocratie. « En un mot, le point de distinction fondamental entre les expériences historiques vietnamiennes et arabes réside dans le fait que la tradition démocratique est totalement absente de cette dernière ${ }^{71}$. »

69 Ibid., p. 145

70 lbid., p. 18-20.

71 ld.
Quarante ans après la rédaction de l'essai de Hafez, Charara, qui s'est longuement entretenu avec son auteur, s'interroge sur la pertinence et la valeur d'une démarche intellectuelle et militante reposant sur un « aveuglement volontaire ${ }^{72}$ ». Peut-on supposer que, en 1976, al-Hafez ignorait tout des abus d'une police politique omniprésente et de l'absence de démocratie au Nord Vietnam? «Qu'est-ce que savoir? » interrogeait Claude Lefort, deux années après la parution de L'archipel du Goulag, au moment où al-Hafez publiait L'expérience historique vietnamienne ${ }^{73}$.

La plurivocité de l'opérateur vietnamien dans les dynamiques de redéfinition des valeurs, $\mathrm{du}$ sens et des enjeux politiques s'organise autour de la tension entre exigence démocratique et violence révolutionnaire, qu'elle soit considérée comme une quasi-valeur ou comme une nécessité déplorée mais inéluctable.

Poursuivre l'étude de ces courants longtemps négligés par les sciences sociales et absents des débats citoyens, permettra de dégager non seulement les traces mais aussi les principes actifs d'une tradition radicale et démocratique arabe, plurielle, différenciée, qui se tisse en dépit de l'imaginaire d'une lutte armée vouée à l'échec, de la transformation

72 Entretien avec l'auteur, 12 juin 2017, Beyrouth.

73 Claude Lefort, Un homme en trop, Paris, Belin, 2015

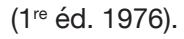


des régimes dits socialistes en dictatures militaires ou à parti unique, et des pratiques coercitives des États qui ont renforcé le conservatisme social dans les sociétés arabes.

« L'esprit démocratique, il ne s'est pas maintenu, mais il était là, nous rappelle Ahmad Beydoun, et l'effort de pédagogie, de conscientisation. Nous aurions dû rester ce que nous étions dans Liban socialiste plutôt que de nous briser dans l'OACL : une école, une école de militants plutôt qu'une formation politique. Ce qui reste valable, c'est ça : une école d'engagement, de fraternité, de démocratie, et cet esprit libertaire dans les rapports interpersonnels aussi bien qu'à l'égard des autorités, n'importe quelles autorités. C'est cela qui constitue une source d'inspiration aujourd'hui ${ }^{74}$.»

74 Entretien avec l'auteur, 9 mai 2017, Amsterdam-Beyrouth. 